\title{
NEUES AUS DER INDUSTRIE
}

\section{Pradovit-Color-Diaselector}

Kleinbildprojektor für die ferngesteuerte Dia-Vorwahl

Technische Fakten

Die Geräte-Einheit Pradovit-Color-Diaselector besteht aus einem modifizierten PradovitColor 250, einem Vorschaltgerät und einem Steuergerät. Das Steuergerät ist ein komplettes Fernbedienungsteil für alle Funktionen des Projektors: ein- und ausschalten, fernfokussieren. Diawechsel vor- und rückwärts. Es erlaubt den ständigen Zugriff zu jedem einzelnen der 50 Dias im Diamagazin. Es ist ein besonderer Vorteil, daß das leichte und kompakte Steuergerät den Vortragsredner nicht an einen bestimmten Platz bindet. Er kann sich damit frei bewegen und die Verlängerungsleitung den Raumverhältnissen anpassen.

Das über das Steuergerät angewählte und das gerade projizierte Dia werden in getrennten Feldern angezeigt. Mit dem Auslöseimpuls erscheint in Sekundenschnelle das gewünschte Dia auf dem Projektionsschirm.

Ist beispielsweise bei der Vorführung Dia 42 erreicht und ein Betrachter bittet, ein vorher gezeigtes Dia zu wiederholen, wird die Wahlkontrollscheibe einfach auf die entsprechende Dia-Nummer, z. B. 36, gestellt und der Auslöseimpuls gegeben. Sofort folgt die Wahlkontrollscheibe diesem numerischen Befehl. Das Magazin fähtt auf dem kürzesten Weg in die angewählte Position und Dia 36 erscheint auf dem Bildschirm.

Für sicheres Ablesen der Dianummern bei verdunkeltem Raum kann eine Skalenbeleuchtung eingeschaltet werden.

$\begin{array}{llcc}\text { Maße } & \text { Projektor } & \text { Vorschaltgerät } & \text { Steuergerät } \\ \text { Länge } & 30 \mathrm{~cm} & 13,5 \mathrm{~cm} & 14 \mathrm{~cm} \\ \text { Breite } & 20 \mathrm{~cm} & 9 \mathrm{~cm} & 11 \mathrm{~cm} \\ \text { Tiefe } & 18 \mathrm{~cm} & 7 \mathrm{~cm} & 6 \mathrm{~cm} \\ \text { Gewicht } & 10 \mathrm{~kg} & 1,1 \mathrm{~kg} & 0,7 \mathrm{~kg}\end{array}$

(Pressedienst der Ernst Leitz GmbH, 633 Wetzlar, Tel. [06441] 292647 u. 292481).

\section{PERSONALIA}

\section{Pappenheim-Preis 1972}

Der Pappenheim-Preis 1972 der Deutschen Gesellschaft für Hämatologie wurde Herrn Dr. Kay Brune, Biozentrum der Universität Basel, für seine Arbeit „Hühnergranulozyten: Ein Modell für die antimikrobielle Funktion peroxydasefreier menschlicher Granulozyten“" verliehen.

\section{ERRATA}

In Heft 6, Bd. XXV, Seite 350, Zeile 6, Leitartikel Ganzoni, ist uns bedauerlicherweise ein Feblet unterlaufen. Es muß heißen:

Dosis $\mathrm{Fe}$ in $\mathrm{mg}=$ Gewicht $(\mathrm{kg}) \times(15-\mathrm{Hb} \mathrm{g} / 100 \mathrm{ml}) \times 2,4+500$. 